\title{
A scalable multi-sink gradient-based routing protocol for traffic load balancing
}

\author{
Hongseok Yoo ${ }^{1}$, Moonjoo Shim ${ }^{1}$ and Dongkyun $\mathrm{Kim}^{2 *}$
}

\begin{abstract}
Wireless sensor networks have been assumed to consist of a single sink and multiple sensor nodes which do not have mobility. In these networks, sensor nodes near the sink dissipate their energy so fast due to their many-toone traffic pattern, and finally they die early. This uneven energy depletion phenomenon known as the hot spot problem becomes more serious as the number of sensor nodes (i.e., their scale) increases. Recently, multi-sink wireless sensor networks have been envisioned to solve the hot spot problem. Gradient routing protocols are known to be appropriate for the networks in that network traffic is evenly distributed to multiple sinks to prolong network lifetime and they are scalable. Each node maintains its gradient representing the direction toward a neighbor node to reach one of the sinks. In particular, existing protocols allow a sensor node to construct its gradient using the cumulative traffic load of a path for load balancing. However, they have a critical drawback that a sensor node cannot efficiently avoid using the path with the most overloaded node. Hence, this paper introduces a new Gradient routing protocol for LOad-BALancing (GLOBAL) with a new gradient model to maximize network lifetime.

The proposed gradient model considers both of the cumulative path load and the traffic load of the most overloaded node over the path in calculating each node's gradient value. Therefore, packets are forwarded over the least-loaded path, which avoids the most overloaded node. In addition, it is known that assigning a unique address to each sensor node causes much communication overhead. Since the overhead increases as the network scales, routing protocols using an address to indicate the receiver in forwarding a packet are not scalable. Thus, GLOBAL also includes an addressing-free data forwarding strategy. Through ns-2 simulation, we verify that GLOBAL achieves better performance than the shortest path routing and load-aware gradient routing ones.
\end{abstract}

Keywords: Wireless sensor networks (WSNs), multi-sink, load balancing, gradient, routing

\section{Introduction}

Traditionally, wireless sensor networks (WSNs) are composed of a large number of sensor nodes and a single sink. Since the distance from each sensor node to a sink (except one-hop neighbor nodes of the sink) is larger than the transmission range of sensor nodes, sensor nodes should transmit their data packet to the sink in a multi-hop manner. Therefore, sensor nodes near the sink tend to dissipate their energy faster than nodes located far away from the sink because they have to forward a large number of data. This uneven energy depletion, known as the hot spot problem [1], drastically reduces

\footnotetext{
* Correspondence: dongkyun@knu.ac.kr

${ }^{2}$ School of Computer Science and Engineering, College of IT Engineering,

Kyungpook National University, Daegu, Korea

Full list of author information is available at the end of the article
}

the lifetime of sensor networks. In addition, as the network scales in terms of the number of sensor nodes, the hot spot problem becomes more serious. Particularly, the WSN architecture with a single sink which creates a many-to-one traffic pattern is considered as the major cause of the hot spot problem [2].

The deployment of multiple sinks might be able to provide a solution to overcoming the architectural limitation of the WSN with a single sink [3]. However, deploying more sinks simply is not enough to solve the problem. We need to design novel communication protocols to realize potential benefits of the multi-sink architecture. When the network traffic is evenly distributed among the multiple sinks, the hot spot problem is expected to be significantly alleviated. Therefore, an efficient routing protocol is required to load balance network traffic 
evenly among the multiple sinks (load-balancing functionality), because the direction of traffic flows is directly determined by the routing protocol. Particularly, in largescale WSNs, communication protocols should scale with the number of sensor nodes [4]. Routing protocols proposed in the literature for WSNs can be categorized in different ways. Depending on the fashion in which routing paths are established, they can be broadly classified into three classes as shown in Figure 1 [5]: (1) proactive, (2) reactive, and (3) hybrid protocols. In proactive protocols, every route is computed in advance, regardless of the need of packet delivery. On the other hand, reactive protocols allow routes to be computed on demand. Hybrid protocols combine properties of both proactive and reactive protocols. Among those protocols, it is known that gradient-based routing protocols which belong to the proactive protocols are scalable, because they are not required to maintain information about network topology [6]. Therefore, we favor a gradient-based routing approach for the large-scale WSNs with multiple sinks. We attempt to mitigate the hot spot problem through load-balancing functionality.

In gradient-based routing protocols, the gradient is defined as the minimum cumulative link (or node) cost along the path, which will be used to transmit its data to the sink. Data from a sensor node flow toward only neighbor nodes having lower gradient. All the gradients of the network nodes create their gradient field whose lowest value the sink has in order to make data always flow toward the sink. The link (or node) cost can take different forms such as hop count, energy consumption, or physical distance, depending on routing design objectives.

Until now, several gradient-based protocols have been proposed to achieve such load balancing [6-11]. They can be categorized into two classes as shown in Figure 1: (a) protocols that exploit the traffic load information of a forwarder's 1-hop neighbor nodes, and (b) protocols that utilize the cumulative traffic load information of sensor nodes over a path from a sensor node to the sink. In the first class, the link cost is defined as the amount of energy consumption or the hop count when establishing the

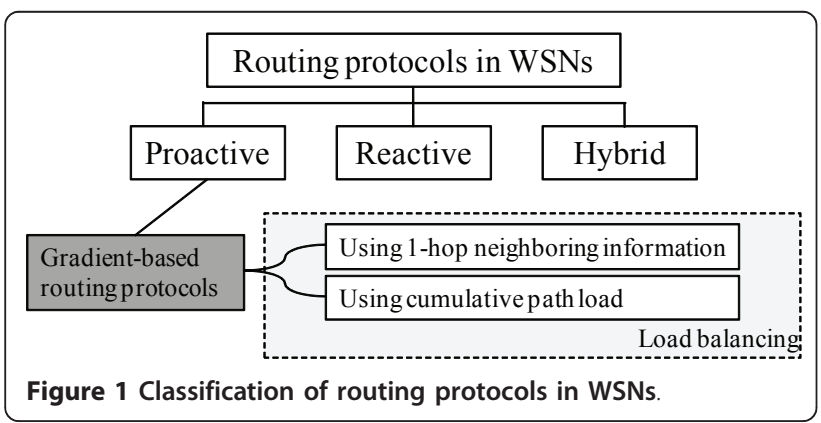

gradient field. Data packets are transmitted along the energy-efficient path to the sink or the path with the minimum end-to-end delivery latency from sensor nodes to the sink. Considering the remaining energy or the queue length of the forwarder's 1-hop neighbor nodes, the neighbor nodes with heavy traffic load or low residual energy are prevented from being selected as a next forwarder, based on their customized forwarding rules. In these protocols, however, sensor nodes cannot spread the traffic evenly since there is no way for them to get the information about heavily loaded region (i.e., hot spot regions near the sinks) several hops away from them [12].

In the second class, each sensor node constructs its gradient using the cumulative traffic load information ${ }^{a}$ of the path potentially used for its data delivery. Then, data flow through the least-loaded path over the established gradient field. Unlike the first class protocols that utilize local information, they can spread the traffic to perform the load balance since they use the cumulative path load. However, they have some drawbacks that a sensor node cannot efficiently avoid using the path with the most overloaded node among its all possible paths since only the cumulative path load is not enough to identify the path including the most overloaded node [13]. We focus on overcoming this drawback in this work.

Based on the above observation, this paper introduces a new gradient-based routing protocol for LOad-BALancing (GLOBAL) in large-scale WSNs with multiple sinks. Assuming that network lifetime is defined as the time elapsed from the deployment to the instant when one of sensor nodes becomes dead, the network lifetime is limited by the lifetime of the most over-loaded sensor node. Therefore, the routing protocol should be able to prevent sensor nodes from using a path including the most overloaded sensor node. In GLOBAL, in order to allow a sensor node to use the least-loaded path which also avoids the most overloaded sensor node, each sensor node calculates its gradient using the weighted average (WA) of the cumulative path load and traffic load of the most overloaded node over the path. The WA technique is a wellknown method used to create a new metric as in [14]. In WA, weights are selected heuristically to obtain overall performance improvements. GLOBAL also includes a heuristic method to determine our weight values.

Generally, an address is used to indicate the receiver in forwarding a packet. Hence, each sensor node should be assigned to its unique address prior to network operations. Such addressing can be made in manufacturing sensor nodes. Since duplicate addresses can exist in the network due to a lot of addressing errors in manufacturing sensor nodes, an automatic addressing protocol is needed to assign a unique address to each node in the network. However, this protocol has much communication overhead as 
well as much energy consumption. In particular, the overhead increases as the network scales. Hence, in gradient routing protocols, a gradient value is simply included in the packet and only neighbor nodes with smaller gradients participate in forwarding the packet. Due to the existence of multiple neighbor nodes with smaller gradients, redundant packet forwarding can occur. Although such redundancy improves reliability, it needs to be suppressed, depending on routing design objectives [15]. GLOBAL, therefore, has an addressing-free data forwarding strategy without such overhead and redundancy.

The rest of the paper is organized as follows. We introduce related works on gradient-based routing protocols with load balance and their general operations in Section II. Section III presents the detailed description for the proposed GLOBAL protocol. Section IV presents the results of the performance evaluation of the proposed scheme and discusses the impact of different values of the simulation parameter on the performance of GLOBAL. Finally, we conclude this paper with future work in Section V.

\section{Related works}

\section{A General operation of gradient-based routing protocols}

In gradient-based routing protocols, no routes are set up prior to sending data. Nodes are assigned only gradients, which are equal to the minimum cumulative link (or node) cost to reach the sink. When a sensor node has some data packets to send, it broadcasts its data packet with its own gradient $G$. Only its neighbor nodes with gradients lower than $G$ participate in rebroadcasting the packet. Gradients of sensor nodes create a gradient field whose lowest value the sink has so that the data packets always flow toward the sink.

A straightforward solution to setting up the gradient field would be found through flooding. Initially, each sensor node sets its gradient to infinity. After the sink broadcasts an ADV (advertisement) packet containing its own gradient 0 , the packet propagates throughout the network. Upon receiving the ADV packet from node M, node $\mathrm{N}$ acquires a path with gradient $G_{M}+C_{N}$, where $G_{M}$ is node M's gradient and $C_{N}$ is the link cost from $\mathrm{N}$ to $\mathrm{M}$. The node $\mathrm{N}$ then compares its current gradient $G_{N}$ and $G_{M}+C_{N}$. If the new gradient is smaller than the old one, it sets $G_{N}$ to $G_{M}+C_{N}$ and broadcasts an ADV packet with an inclusion of its new gradient. Whenever a sensor node receives an ADV packet with gradient smaller than its current gradient, it updates its gradient accordingly and broadcasts a new ADV packet.

\section{B Gradient-based routing protocols with load balancing}

In this section, two classes of existing gradient-based routing protocols with load balancing which are mentioned in
Section I are described in more detail. We briefly review representative protocols belonging to each class.

\section{B.1 Using 1-hop neighboring information}

Huang et al. [6] proposed the SGF protocol. Since SGF aims to route data along an energy-efficient path to the sink, energy consumption is used as link cost for establishing a gradient of each sensor node. Assuming that the transmission power of sensor nodes is adjustable, each sensor node (say node $i$ ) measures the actual channel gain $\delta_{i j}$ based on its transmission power and received power of a control packet (i.e., ADV packet) sent by its neighbor node (say node $j$ ) and calculates the minimum transmission power required to send data from node $i$ to node $j$, from $\delta_{i j}$.

In order to balance network traffic load, SGF introduces a new forwarding method that gives sensor nodes with more remaining energy more chance to forward their neighbor nodes' packets. Hence, node $i$ transmits its data with its gradient, and then, when node $j$ receives a packet from its neighboring node $i$, node $j$ determines whether to forward the received packet, based on its residual energy as well as gradients of node $i$ and itself. Some protocols are similar to SGF (see $[7,8])$.

\section{B.2 Using cumulative path load}

Lie et al. [9] proposed the PWave protocol, which takes its inspiration from potential theories and principles in resistive electric networks. In PWave, a gradient field is constructed in a WSN and routing is determined by the gradients of nodes just like the way that an electric current flows in a resistive electric network. The sink's gradient is set to 0 , and the others' gradients are assigned to minimize a certain global objective function. Data can only flow from each sensor node toward its neighbor nodes having lower gradients than it has. As stated in [9], by minimizing the global objective function, PWave yields a multi-path proportional routing scheme where the sensor node spreads its traffic across multiple paths inversely proportional to the cumulative path cost. The cumulative path cost is defined as the sum of link costs along the path.

The authors of PWave argued that the link cost can take different values according to different routing design objectives. If the link cost is set to 1 , data flows are routed based on path hop counts. For another example, they suggested that if the link cost is set to the reciprocal sum of the residual energy of sensor nodes constructing a link, PWave can maximize network lifetime. We found that PWave can maximize the sum of the lifetime of sensor nodes from their mathematical derivation. However, assuming that network lifetime is the time elapsed from the deployment to the instant when one of sensor nodes becomes dead, it is essential to extend the lifetime of the most overloaded sensor node in the network, because the network lifetime is limited by the most overloaded sensor 
node. Similar approaches based on the cumulative path load are founded in $[10,11]$.

\section{GLOBAL: a gradient-based routing protocol for LOad-BALancing}

The proposed GLOBAL protocol is designed under the following assumptions.

- Types of traffic: In our target applications, both periodic traffic and aperiodic event-driven traffic are generated from sensor nodes.

- Position of sinks and sensor nodes: The sinks and sensor nodes are randomly placed, and all sinks and sensor nodes have no mobility.

- Communication ranges: The fixed and homogeneous transmission range is set, and the carrier sensing, interference range is two times larger than the transmission range.

- Security concern: As stated in [16], since the hardware of sensor nodes is not tamper-resistant, the attacker can control any sensor node through physical compromise and it can also acquire the secret keys to participate in legitimate inter-node communications. Even though some cryptographic protocols can be applied to inter-node communications, the attacker can still access the cryptographic keys through physical compromise in order to participate in the communications in a legitimate way. After getting control of a sensor node, the attacker can force some sensor nodes to perform malicious attacks (i.e., altering/dropping the messages which should be forwarded to the next-hop node). In this current version of GLOBAL, the countermeasure for such attacks are not included, since security issues are beyond the scope of this work.

The GLOBAL protocol consists of three phases: gradient field establishment, data forwarding, and gradient field maintenance phases. In the gradient field establishment phase, sinks flood an ADV packet. In receiving an ADV packet, a senor node calculates its gradient using the information specified in the received ADV packet. In the data forwarding phase, an address is useless to indicate the receiver in forwarding a packet. When a sensor node updates its gradient, it acquires the gradient value of its next-hop node. Hence, the gradient value can be used as the next-hop node identifier. In the gradient field maintenance phase, since the traffic load distribution of sensor nodes changes dynamically after sensor nodes start to transmit their data, each sensor node refreshes its gradient whenever it overhears a periodic data packet from its neighbor nodes. The periodic data packet includes the information required to calculate the gradient value. In the rest of this section, we first introduce the proposed cost \& gradient models and then present the detailed description of each phase in the proposed GLOBAL protocol.

\section{A The proposed cost \& gradient models \\ A.1 The cost model}

The link (or node) cost that is used to evaluate routes is a very important component of the gradient-based routing protocols. For load balancing, GLOBAL uses traffic load of sensor nodes as cost for gradient construction (Detailed mechanisms of gradient construction are given in the next subsection). Different from the value which is defined at the link level such as the amount of energy consumed in transmitting a packet and the geographical distance between sensor nodes, a traffic load is the cost defined at the node level.

As indicated in [17], the traffic load $T L_{i}$ in each sensor node (say node $i$ ) is defined as the sum of the traffic passing through node $i$ and the traffic passing through node $i$ 's neighbor nodes due to the broadcast nature of radio channels. Although $T L_{i}$ can be calculated based on the actual amount of traffic, the amount of local energy dissipated in the radio communication can be utilized to obtain $T L_{i}$ since all the communication activities (transmission, reception, and overhearing) occurring at node $i$ and node $i$ 's neighbor nodes result in decrease in node $i$ 's residual energy [18]. In addition, let us assume that two sensor nodes (say nodes $A$ and $B$ ) consume the same amount of energy per unit time and their energy expenditure only comes from the communication activities, but node $B$ has the larger amount of residual energy than node $A$. In this case, although they consume the same amount of energy for the communication activities, node $A$ eventually dies earlier than node $B$. Therefore, a traffic load of node $B$ should be estimated lower than node $A$ to make other nodes favor node $B$ than node $A$ as their forwarder. Based on the above observation, we use the residual energy depletion rate (REDR) as the metric that measures the traffic load of a given sensor node. In this work, we assume that all sensor nodes are battery powered and the amount of their energy consumed in executing activities (i.e., sensing) except for communication activities is identical to each other. Therefore, REDR is only affected by the energy consumption resulting from the communication activities. Hence, REDR can be substituted for traffic load.

In GLOBAL, node $i$ updates its gradient whenever it receives an ADV or a periodic data message from its neighbor nodes (Refer to following sub-sections for details). Before updating its gradient, node $i$ first updates its REDR in order to use the latest REDR information. Whenever node $i$ has received the ADV or periodic data message from its neighbor nodes at time $a, \operatorname{REDR}_{i}^{\text {sample }}$ is calculated by Equation 1, where $e_{i}^{a}$ and $e_{i}^{b}$ are the amount 
of residual energy at times $a$ and $b$, respectively. $b$ indicates the time when node $i$ previously received the ADV or periodic data message. We assume that each sensor node can measure its residual energy through a battery monitoring system ${ }^{\mathrm{b}}$ [19]. $\mathrm{REDR}_{i}^{\text {sample }}$ represents the depletion rate of residual energy during the last $a-b$ seconds.

$$
\operatorname{REDR}_{i}^{\text {sample }}=\left(1-\frac{e_{i}^{a}}{e_{i}^{b}}\right) /(a-b)
$$

Upon obtaining a new $\operatorname{REDR}_{i}^{\text {sample }}$, node $i$ updates its residual energy depletion rate $\operatorname{REDR}_{i}$ through the wellknown exponential weighted moving average method to mitigate the fluctuation of $\operatorname{REDR}_{i}^{\text {sample }}$ (see Equation 2). In Equation 2, $\mathrm{REDR}_{i}^{\text {old }}$ represents the previous REDR value of node $i$ and is the smooth weighing factor.

$$
\operatorname{REDR}_{i}=\alpha \cdot \operatorname{REDR}_{i}^{\text {old }}+(1-\alpha) \cdot \operatorname{REDR}_{i}^{\text {sample }}
$$

To better reflect the current status of energy dissipation of sensor nodes, we give higher weight to the new $\mathrm{REDR}_{i}^{\text {sample }}$ by setting $\alpha$ to 0.3 . Although sensor nodes do not have data to relay, they have to send packet periodically. Therefore, an initial value of $\mathrm{REDR}_{i}$ is taken according to the amount of periodic data at the node $i$.

\section{A.2 The gradient model}

GLOBAL allows sensor nodes to set their gradients with two design goals as follows.

- Sensor nodes should favor a path which is less loaded.

- Sensor nodes should avoid a path including the most overloaded sensor node.

If only the first design goal is considered, the gradient of node $i$ (denoted by $G_{i}$ ) should be set to the sum of the REDR values of the sensor nodes, which constitute the path potentially used for data delivery. Therefore, assuming that the path of node $i$ consists of $n$ sensor nodes including node $i, G_{i}$ is calculated by Equation 3, where $\mathrm{REDR}_{j}$ is the REDR value of the $j$ th sensor node over the path. However, with the gradient model driven by Equation 3, a sensor node cannot efficiently avoid using the path with the most overloaded node among its all possible paths since only the cumulative path load is not enough to identify the path including the most overloaded node [13]. Thus, Equation 3 should be revised to achieve the above two goals.

$$
G_{i}=\sum_{j=1}^{n} \operatorname{REDR}_{j}
$$

If $G_{i}$ is calculated by Equation 4, sensor nodes can avoid the path including the most overloaded node. However, $\max _{1 \leq j \leq n} \mathrm{REDR}_{j}$ does not increase as the path has more hops. Hence, it is not an useful gradient value as explained in Section II.A. Therefore, in order to meet the two design goals, we attempt to combine the desirable properties of the two values obtained by Equations 3 and 4 . Therefore, their weighted average is taken by adopting the method introduced in [14]. See Equation 5.

$$
G_{i}=\max _{1 \leq j \leq n} \operatorname{REDR}_{j}
$$

Assuming that $\mathrm{REDR}_{n}$ is $\max _{1 \leq j \leq n} \mathrm{REDR}_{j}$, Equation 5 can be changed to Equation 6 . Consequently, $G_{i}$ indicates the weighted sum of the REDR values of the sensor nodes which constitute the path. The REDR of the most overloaded sensor node is weighted differently from the REDRs of the other sensor nodes over the path, according to Equation 6.

$$
\begin{aligned}
& G_{i}=\beta \sum_{j=1}^{n} \operatorname{REDR}_{j}+(1-\beta) * \max _{1 \leq j \leq n} \operatorname{REDR}_{j} \\
& G_{i}=\beta \sum_{j=1}^{n-1} \operatorname{REDR}_{j}+\operatorname{REDR}_{n}
\end{aligned}
$$

In Equations 5 and 6, $\beta$ is a weighting factor of both parameters and ranges between 0 and 1 . In Section IV. $\mathrm{B}$, we will investigate the impact of $\beta$ on the GLOBAL's performance using ns-2 simulation and introduce a new heuristic method to choose $\beta$, based on observations from simulation results.

\section{B Gradient field establishment}

GLOBAL utilizes the naive flooding-based gradient field establishment algorithm to set up initial gradients of sensor nodes ${ }^{\complement}$. When a network is initialized, each of sinks floods an ADV packet one after another with a pre-defined time interval, which is set to enough time to ensure that two consecutive floodings do not interfere with each other. The ADV packet contains the following three fields for the gradient field construction. Initially, sinks have the three fields whose values are 0 . For example, in Figure 2, when sensor node B rebroadcasts the ADV packet received from sensor node A, hcnt, sum_redr, and max_redr carry $2, \mathrm{REDR}_{A}+R E D R_{B}$ and $\max \left(\operatorname{REDR}_{A}, \mathrm{REDR}_{B}\right)$, respectively. $\mathrm{REDR}_{A}$ and $\mathrm{REDR}_{B}$ represent the REDR values of sensor nodes $A$ and $B$, respectively.

- hcnt: The number of hops (or links) that the ADV packet has traversed. 


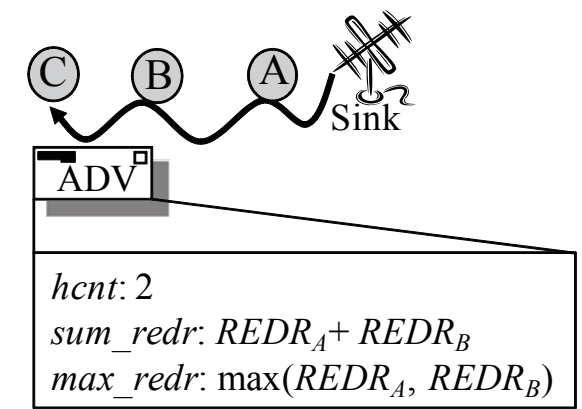

Figure 2 The flooding of ADV.

- sum_redr: The sum of REDR values of sensor nodes that the ADV packet has traversed.

- max_redr: The maximum REDR value among REDR values of sensor nodes that the ADV packet has traversed.

When a sensor node (say node $i$ ) initially receives an ADV packet, it firstly initializes all local variables to NULL and its local variables including its gradient are updated through the fields specified in the received ADV packet (see Algorithm 1). When node $i$ receives an ADV packet for the first time, it increases the hcnt value appeared in the ADV packet by one in order to account for the new hop distance. $s_{-} h c n t$ keeps the increased value (i.e., $h c n t+1)$. Since node $i$ initially does not have any path information, the newly acquired path will be used to transmit data as the shortest hop path. Hence, path_hcnt is set to the same value of $s_{-}$hcnt. Both hopcount variables are used to avoid an excessive increase in the path length as followings. Then, after updating its $\mathrm{REDR}_{i}$ through the procedure described in Section III. A.1, node $i$ updates sum_redr $r_{L}$ and max_redr $r_{L}$ as shown in Algorithm 1. sum_redr $r_{L}$ and max_redr $r_{L}$ indicate the cumulative path load and traffic load of the most overloaded node over the path, respectively. Using Equation 7 , node $i$ calculates its gradient $G_{i}$ and maintains $G_{i}$ in its local memory.

From the proposed gradient model introduced in Section III.A.2, $G_{i}$ is calculated by averaging the two metrics, namely sum_redr $r_{L}$ and max_redr $r_{L}$, where $\beta$ is a weighting factor of both parameters and ranges between 0 and 1 . After updating its local variables, node $i$ updates the fields in the ADV packet from its local variables as follows: hcnt $=$ path_hcnt, sum_redr $=$ sum $_{-} r e d r_{L}$, max ${ }_{-} r e d r=$ max ${ }_{-}-$ redr $_{L}$. Finally, it rebroadcasts the ADV packet.

$$
G_{i}=\beta \cdot \text { sum_redr } r_{L}+(1-\beta) \cdot \max \_r e d r_{L}
$$

After acquiring the initial path, node $i$ may still discover a less loaded path than the initial path when it receives a duplicate ADV packet. However, since the latency of an end-to-end delivery increases as the number of hops in the path increases, it is not desirable to select a very long path with respect to load balancing. Therefore, GLOBAL defines a system parameter $K$ in order to prevent a sensor node from selecting an extremely long path. Given paths providing lower gradients than a current gradient of node $i$, GLOBAL allows node $i$ to designate only the path whose hop count is not larger than $s_{-} h c n t+K$ as its new path. To do so, when node $i$ receives a duplicate ADV packet, it first calculates the gradient $g$ of the newly discovered path after updating its REDR using Equation 8.

$$
g=\beta \cdot\left(\text { sum_redr }+\operatorname{REDR}_{i}\right)+(1-\beta) \cdot \max \left(\text { max_redr, } \operatorname{REDR}_{i}\right)
$$

Only if $g$ is lower than $G_{i}$ and $h c n t$ is smaller than $s \_h c n t+K$, node $i$ designates the newly discovered path as its new path. Then, it sets $g$ to $G_{i}$ and rebroadcasts the ADV packet after updating fields in the ADV packet as mentioned before. Particularly, whenever node $i$ receives a duplicate ADV packet, it updates the $s_{-} h c n t$ value if $h c n t$ of the received packet is smaller than a current s_hcnt value - 1 .

\section{Data forwarding with the proposed addressing-free forwarding strategy}

GLOBAL includes an addressing-free data forwarding strategy. In GLOBAL, if a node (say node A) updates its gradient after receiving a packet, the node from which the packet is received should be designated as the node A's next-hop node. Instead of using an address for forwarding packets, GLOBAL uses the gradient value as the next-hop node identifier. Suppose that a node (say

Algorithm 1 Updating local variables at node $i$

s_hcnt : the shortest number of hops to reach a sink from node $i$.

path_hcnt : the number of hops of the current path which node $i$ transmits data over.

sum_redr $r_{L}$ : the sum of REDR of nodes (including node $i$ ) along the current path which node $i$ transmits data over.

max_redr $r_{L}$ : the maximum REDR value among REDR values of sensor nodes (including node $i$ ) along the current path which node $i$ transmits data over.

$G_{i}$ : node $i$ 's gradient.

if (ADV is received for the first time) then

$$
\begin{aligned}
& \text { s_hcnt }=h c n t+1 \\
& \text { path_hcnt }=s \_h c n t \\
& \text { update REDR } \\
& \text { sum_redr } r_{L}=\text { sum_redr }+\mathrm{REDR}_{i} \\
& \text { if }\left(\mathrm{REDR}_{i}>\text { max_redr }\right) \text { then } \\
& \quad \text { max_redr } r_{L}=\mathrm{REDR}_{i} \\
& \text { else } \\
& \quad \text { max_redr } r_{L}=\text { max_redr }
\end{aligned}
$$




\section{end if}

update $G_{i}\left(=\beta \cdot\right.$ sum_redr $\left._{L}+(1-\beta) \cdot \max \_r e d r_{L}\right)$

else if (Duplicate reception of ADV, or overhearing of a data packet) then update $\mathrm{REDR}_{i}$

calculate $g\left(=\beta \cdot\left(\right.\right.$ sum_redr $\left.+\operatorname{REDR}_{i}\right)+(1-\beta)$. $\left.\max \left(\max r e d r, \mathrm{REDR}_{i}\right)\right)$

if $\left(g<G_{i}\right.$ \&hcnt $\left.<\left(s_{-} h c n t+K\right)\right)$ then

$G_{i}=g$

sum_redr $r_{L}=$ sum_redr $+\mathrm{REDR}_{i}$

if $\left(\mathrm{REDR}_{i}>\right.$ max_redr) then

max red $r_{L}=\mathrm{REDR}_{i}$

else

max_red $r_{L}=$ max_red $r$

end if

end if

if $h c n t<s_{-} h c n t-1$ then

s_hcnt $=h c n t+1$

end if

end if

node $j$ ) had been the next-hop node of a node (say node $i$ ), based on their previous gradient values. Note that the gradient of each node can be changed over time. Hence, after node $i$ acquired node $j$ 's gradient as its nexthop identifier, node $j$ should still maintain the gradient value of which it informed $i$ even though node $j$ has a new gradient value, so that node $j$ can relay the packet forwarded from node $i$. In GLOBAL, each node broadcasts the packet with its current gradient $G_{\text {nexthop }}$ and maintains that gradient value in local memory $G_{\text {informed }}$. When a node updates its gradient after receiving the packet, it uses $G_{\text {nexthop }}$ as the next-hop node identifer when sending next packets. A node receiving a packet decides to forward the packet only if $G_{\text {nexthop }}$ of the received packet is equal to its local $G_{\text {informed }}$ value.

\section{Gradient field maintenance}

The initial gradient field becomes inaccurate, since the traffic load distribution of sensor nodes varies dynamically after sensor nodes start to transmit their data. In addition, some sensor nodes may fail to receive an ADV message since wireless channel is vulnerable to data loss. Therefore, they cannot calculate their gradients. This requires the gradient field to be refreshed timely in order to avoid the uneven traffic load distribution as well as to enable sensor nodes to acquire their accurate gradients.

Most of gradient routing protocols rely on the periodic flooding of ADV packet to reconfigure gradient fields $[4,15]$. However, since such a flooding incurs excessive packet overhead, the network becomes stressed, which is not scalable. Hence, GLOBAL has gradients to be updated during data transmissions without any dependency on flooding, which leads to little overhead. In our target application scenario, sensor nodes transmit data in a periodic manner. All periodic data packets include three fields specified in the ADV packet. Whenever a sensor node (say node $i$ ) overhears a data packet from its neighbor node except its current next-hop node (say node $j$ ), they process the data packet just as they process a duplicately received ADV packet. In order to prevent the node $j$ for designating node $i$ to its next-hop node, node $j$ does not update its gradient when it receives a data packet from node $i$. As explained in Section III.C, each sensor node advertises its gradient value as the identifier of a next-hop node (instead of address) to its neighbor nodes and it can also acquire a new identifier when a gradient is updated. Hence, when node $i$ receives a packet from node $j$, the packet should carry the gradient value of which node $j$ informed node $i$ previously in order to allow node $i$ to find out whether the received packet has been sent from node $j$ or not. Therefore, in GLOBAL, each node broadcasts its packet with the current $G_{\text {informed }}$ value. When a sensor node receives a packet from a neighbor node, it can find out whether the received packet has been sent from its next-hop node or not by checking whether $G_{\text {nexthop }}$ is equal to $G_{\text {informed }}$ in the received packet. In particular, $G_{\text {nexthop }}$ of a sensor node is set to NULL (as mentioned in Section III.B), because it cannot have the information on the current next-hop node until it receives an ADV message. Hence, a sensor node failing to receive an ADV message can acquire its gradient once it overhears a periodic data packet from its neighbor nodes.

After a senor node (say node $i$ ) decides its path, its traffic load through the path keeps changing over time. Hence, node $i$ 's gradient should be dynamically updated accordingly. Therefore, when node $i$ overhears a data packet from its current next-hop node, it first checks the hop count of the path. If hcnt is not larger than $s_{-} h c n t+$ $K$, it updates its gradient in order to reflect the changes of traffic load over the path which it is currently using. Otherwise, node $i$ sets its gradient to infinity and tries to find a new path whose hop count is not larger than $s_{-}$hcnt $+K$. In addition, when node $i$ fails to overhear a periodic packet from its current next-hop node (say node $j$ ), the $G_{\text {nexthop value maintained in node } i \text { may become unequal }}$ to the $G_{\text {informed }}$ value maintained in node $j$. In this case, node $i$ should not designate node $j$ as its next-hop node. Thus, node $i$ sets its gradient to infinity and tries to find a new path when it fails to receive node $j$ 's periodic packet. We assume that each node knows the transmission interval of periodic traffic.

\section{Performance evaluation}

\section{A Simulation setup}

In this section, we evaluate the performance of GLOBAL in grid networks ${ }^{\mathrm{d}}$ using the ns-2 simulator. To create grid networks, we constructed $N \times N$ square grids where a distance between adjacent nodes in each row and column is 
$20 \mathrm{~m}$. Since the hot spot problem becomes more serious as the network scales, we tested different network scales (i.e., $N=20,30,40)$. Three sink nodes were positioned in the farthest locations among each other in the grid, and the others were configured as sensor nodes. Transmission, interference, and carrier sensing ranges were set to 35,70 , and $70 \mathrm{~m}$, respectively. Each sensor node generated its periodic traffic as well as event-driven one. $R \%$ of nodes generated the event-driven traffic, and they were randomly re-selected every $10 \mathrm{~s}$. We assumed that the event-driven traffic was also periodic for the simplicity of simulations, but it had shorter transmission interval. In addition, different $R$ values (i.e., $R=5,10,15$ ) were used to vary traffic rates.

We compared GLOBAL with two protocols, SPR (shortest-hop path routing) and CPL (cumulative path load). In SPR, each node transmits its data using the shortest path without any consideration of load balancing. On the other hand, in CPL, each node constructs its gradient based on the cumulative traffic load of a path. If $\beta$ is set to 1 , GLOBAL works in the same as CPL. Through performance comparisons of GLOBAL with CPL, we could observe the superiority of the proposed gradient model that allows each sensor node to calculate its gradient by considering both the cumulative path load and the most overloaded node over the path.

As an energy consumption model, we employed the energy model used in [12]. The packet transmission and reception energy consumption were set to $50 \mathrm{~nJ} * l+100$ $\mathrm{pJ} / \mathrm{bit} / \mathrm{m}^{2} * l * d^{2}$ and $50 \mathrm{~nJ} * l$, respectively, where each of $l$-bit packets is transmitted at a distance $d$. From the assumption of a fixed transmission range, $d$ was set to $30 \mathrm{~m}$. In addition, due to the broad-cast nature of the wireless channel, a sender's neighbor nodes overhear its transmissions even if they are not its intended receivers. As mentioned in [20], a receiver cannot decide whether or not it is an intended receiver until the entire packet is received. Therefore, such overhearing causes unintended receivers to waste energy. Hence, assuming that the energy consumption caused by additional processing of the packet header is negligible, GLOBAL does not require additional energy consumption caused by overhearing. Certainly, if the receiver has a mechanism to decode the packet header alone and then turn off the radio during the period of receiving the remaining part of the packet for an unintended recipient, the additional energy savings can be expected. However, such sophisticated scheduling comes from a high price in terms of hardware complexity and may induce delays in processing at higher layers. In particular, such scheduling with high complexity cannot be easily realized in a resourceconstrained sensor node. Nevertheless, if we assume that such scheduling can be applied to sensor nodes with a low price, the amount of additional energy consumption caused by overhearing in GLOBAL is required only in receiving the additional three header fields, hcnt, sum_redr, and max_redr (Their total size for the three fields is 5bytes.). In our simulations, a packet overhearing requires GLOBAL and CPL to consume more additional energy in receiving the three fields, as compared to SPR. The initial energy of sensor nodes was set to be $1 J$. Other simulation parameters are summarized in Table 1.

We define four performance metrics as follows.

- Balance factor $(\mathrm{BF})$ : We measure the balance factor of traffic load of sensor nodes. BF is utilized to investigate how well the traffic load is balanced across sensor nodes [21]. Let $L_{i}$ be the traffic load of a sensor node $i$. BF with the $n$ number of nodes is defined as Equation 9. Under the configurations where traffic conditions applied to simulations of each routing protocol are different from each other, measuring their $\mathrm{BF}$ values looks unfair. In our simulations, we therefore investigated the evolution of $\mathrm{BF}$ till the lifetime of a network where SPL is used as its underlying routing protocol. SPL is the routing protocol showing the lowest network lifetime. Since the network lifetime of SPL varies depending on network scales, Figures 3 and 4 show different $\mathrm{x}$-axis ranges accordingly.

$\mathrm{BF}=\frac{\left(\sum_{n}^{i=1} L_{i}\right)^{2}}{n \sum_{n}^{i=1} L_{i}{ }^{2}}$

- Network lifetime: Network lifetime can be defined variously according to a type of applications [22]. In GLOBAL, we define the network lifetime in two ways: (a) the time from network deployment to the instant when one of any sensor nodes dies, denoted by $L T_{1}$, and (b) the time from network deployment to the instant when $P$ percentage of sensor nodes

Table 1 Simulation parameters

\begin{tabular}{ll}
\hline Parameter types & Value \\
\hline Simulation duration time & $600 \mathrm{~s}$ \\
Data packet size & 100 Bytes \\
Tx interval of periodic traffic & $10 \mathrm{~s}$ \\
TX interval of event-driven traffic & $1 \mathrm{~s}$ \\
IFQ length & 10 \\
MAC protocol & CSMA/CA mode of IEEE 802.15.4 \\
Data rate & 2 Mbps \\
Propagation model & Two-ray ground \\
\hline GLOBAL & \\
$\alpha$ & 0.3 \\
$K$ & 5 \\
\hline
\end{tabular}




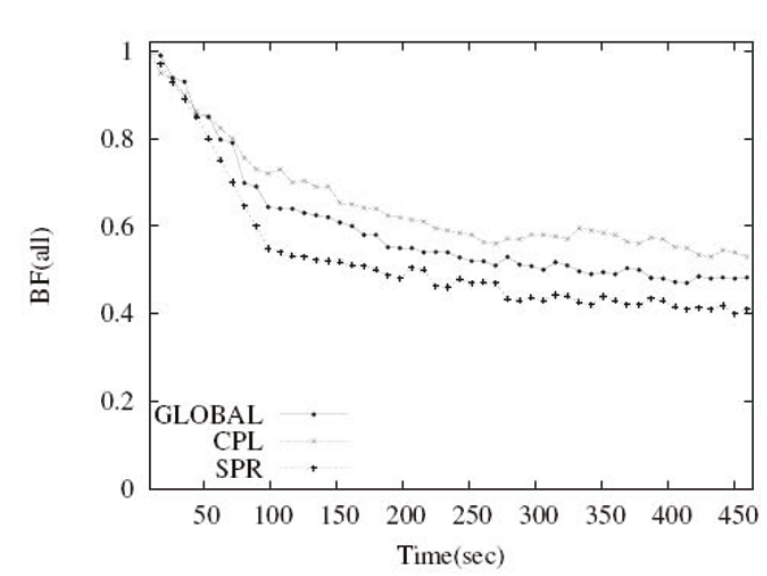

(a)

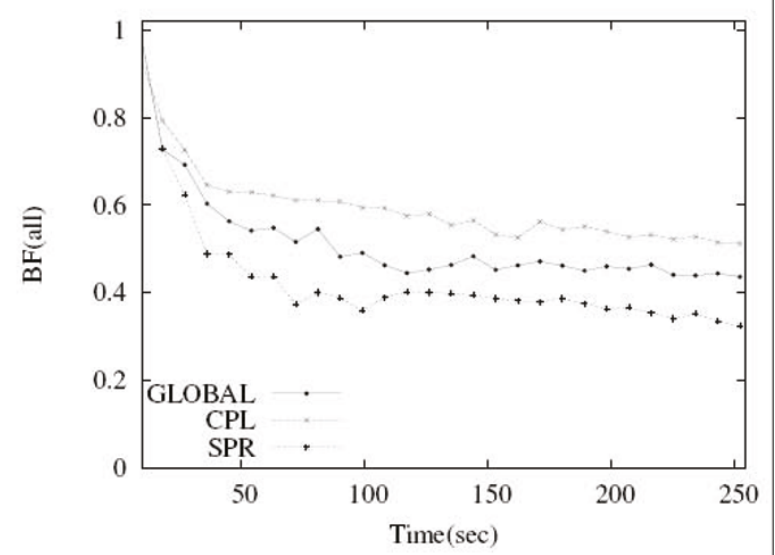

(b)

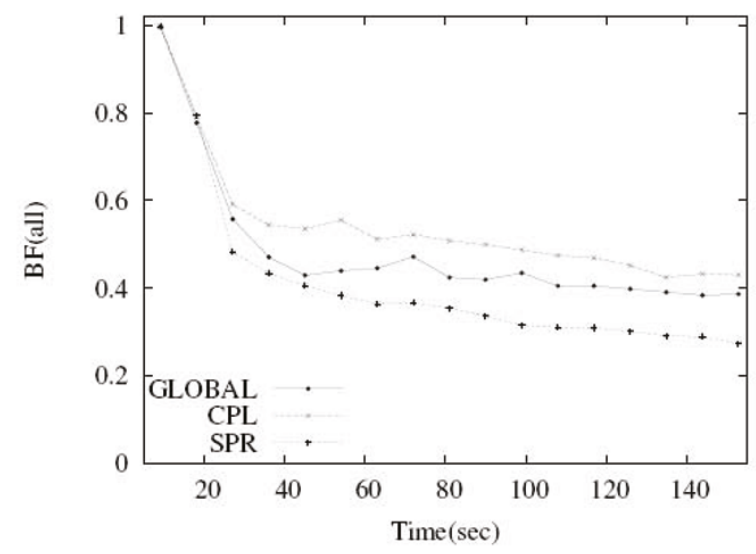

(c)

Figure 3 Evolution of $\mathbf{B F}(\mathbf{a l l})$. a $N=20,400$ nodes, $\mathbf{b} N=30,900$ nodes, $\mathbf{c} N=40,1,600$ nodes.

die, denoted by $L T_{\%}$. In particular, the $P$ values are varied in our simulations.

- Packet delivery ratio (PDR): PDR is defined as the ratio of the total number of data packets received by sinks to the total number of data packets transmitted by sensor nodes.

- Average end-to-end delay: The end-to-end delay is averaged over all surviving data packets from sensor nodes to sinks.

First, we investigate the impact of $\beta$ on the performance of GLOBAL in the following section. We also design a heuristic method to choose $\beta$ based on observations from simulation results. We introduce simulation results for each of the above-mentioned metrics, and the analysis of routing control overhead in the protocols is presented in Section IV.C
B The impact of $\beta$ and a heuristic method to choose $\beta$ As shown in Equation 5, $\beta$ is tunable. $\beta$ determines the shape of the gradient field, which determines GLOBAL's routing operations. A larger $\beta$ value enables each sensor node to more favor the least-loaded path, while a smaller $\beta$ one makes the path excluding the most overloaded node more favored. The network traffic is more evenly distributed as the least-loaded path is preferred to use. However, a decrease in network lifetime $\left(L T_{1}\right)$ is unavoidable since only the cumulative path load is not enough information to identify the path including the most overloaded node. Therefore, there exists a trade-off between the even distribution of traffic load and network lifetime. To explore the impact of $\beta$ on the GLOBAL performance, we evaluated the GLOBAL's performance with various $\beta$ values. We measured $B F$ for all sensor nodes (denoted by $\mathrm{BF}($ all $)$ ) in the grid scenario where $N$ is set 


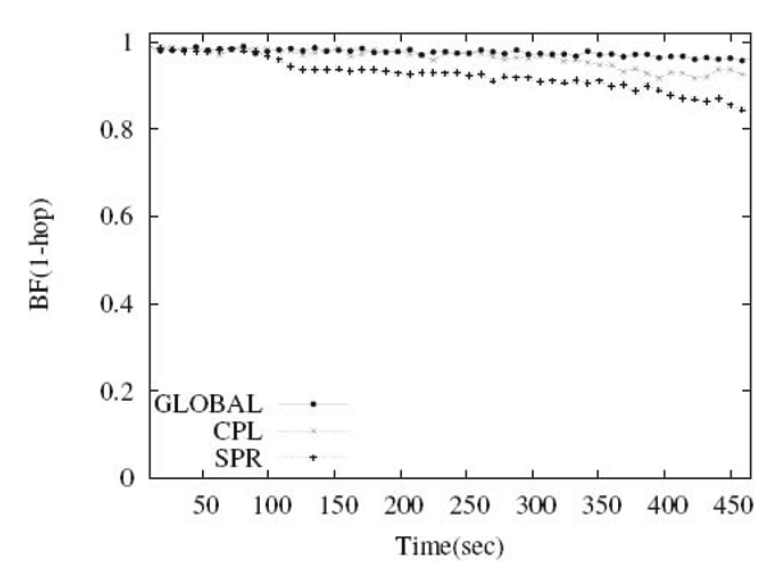

(a)

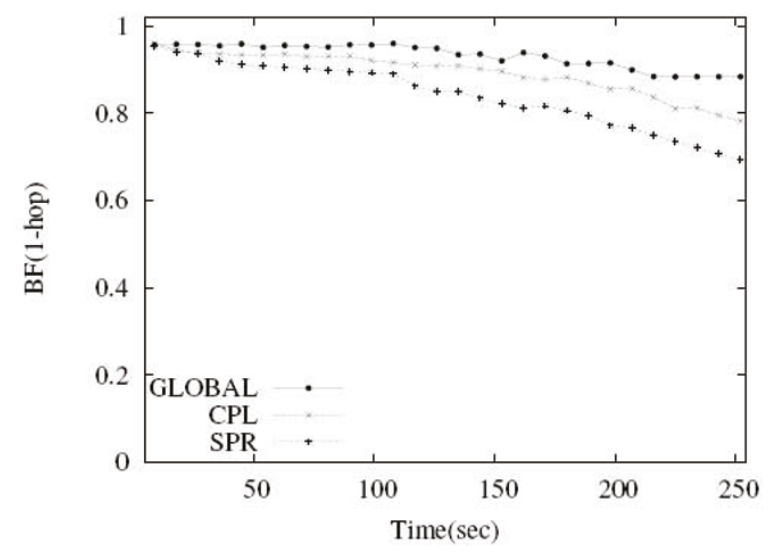

(b)

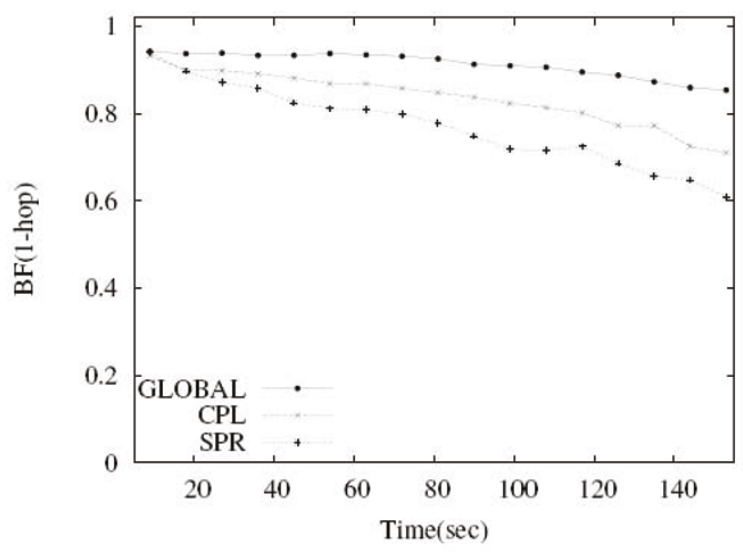

(c)

Figure 4 Evolution of $\mathbf{B F}(1$-hop). a $N=20,400$ nodes, $\mathbf{b} N=30,900$ nodes, $\mathbf{c} N=40,1,600$ nodes

to 20 . As expected, a larger $\beta$ value makes the $\mathrm{BF}($ all $)$ value for traffic load of sensor nodes to increase, but it makes the network lifetime to decrease as shown in Figure 5. As mentioned in Section III.A.2, setting $\beta$ to 1 does not make sense, since the gradient value is not guaranteed to increase with path length.

We also captured the load distribution of network traffic in the 20x20 grid network with two sinks when SPR was used as a underlying routing protocol. As shown in Figure 6, due to the many-to-one traffic pattern (i.e., convergecast traffic to each sink), sensor nodes around sinks tend to be highly overloaded. Since the network lifetime is limited by the lifetime of the most overloaded node among them, the selection of a value should allow traffic to make a detour around the most overloaded node, especially around sinks.

Based on the above observations, we introduce a heuristic method to choose $\beta$ in order to maximize the network lifetime while minimizing the decrease in $\mathrm{BF}(\mathrm{all})$. In GLOBAL, $\beta=\frac{s_{-} \text {hcnt }}{\text { net-diameter }}$, where $s_{-}$hcnt is the shortest number of hops to reach a sink from a sensor node (see Algorithm 1) and net - diameter is the maximum number of hops from one end of the network to the other ${ }^{e}$. Through the proposed heuristic method, each sensor node is enabled to more favor paths excluding the most overloaded node as its shortest hop distance to a sink decreases. This makes the traffic load of sensor nodes around sinks effectively balanced while allowing sensor nodes far away from sinks to favor the least-loaded path.

Here, for the purpose of demonstrating the effectiveness of the proposed heuristic method, we measured BF (all) and network lifetime $\left(L T_{1}\right)$ of GLOBAL under the same simulation configurations as mentioned above (see Figure 7a). As shown in Figure 7b, GLOBAL shows the best network lifetime with a negligible deterioration in $\mathrm{BF}($ all $)$. 


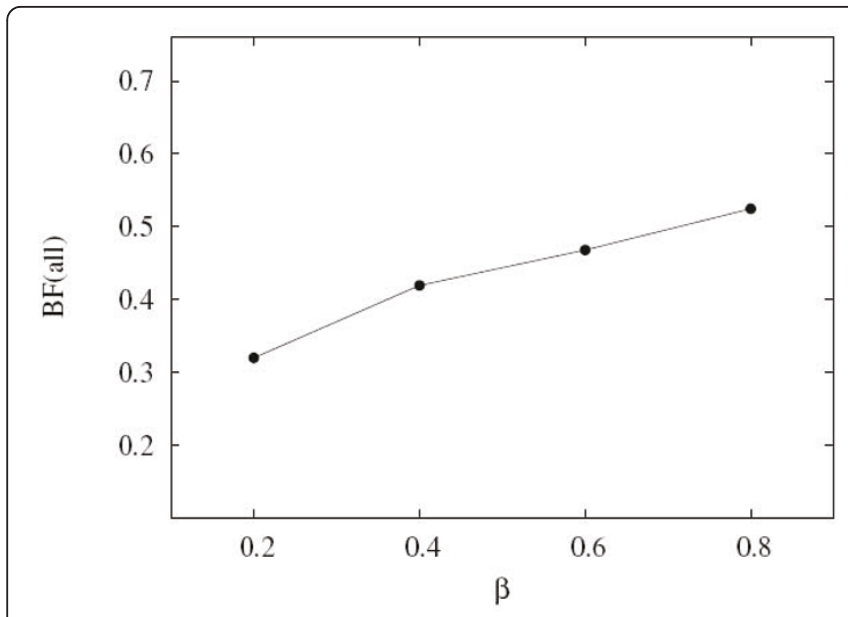

(a)

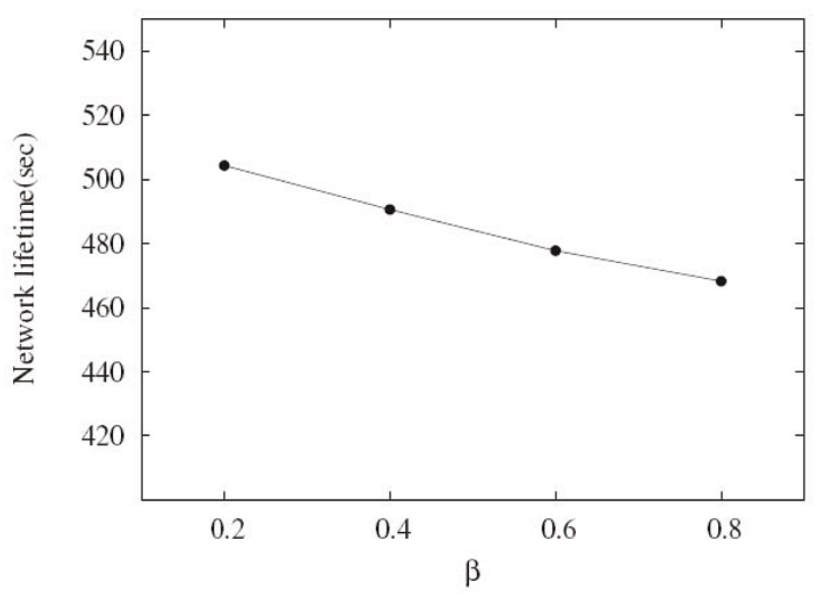

(b)

Figure $5 \mathrm{BF}($ all $)$ and network lifetime for different $\boldsymbol{\beta}$ values. a $\mathrm{BF}\left(\right.$ all). b Network lifetime $\left(L T_{1}\right)$.

\section{Performance results}

\section{C.1 BF}

First, for each network scale, we measured the BF(all) value of three compared protocols (GLOBAL, SPR, CPL). $R$ was set to $5 \%$ in these simulations. Figure 3 shows the evolution of $\mathrm{BF}(\mathrm{all})$ for each network scale. At an early stage of the simulation, all the protocols have $\mathrm{BF}(\mathrm{all})$ of almost 1 because sensor nodes cannot generate enough traffic to make traffic load distribution unbalanced. For all network scales, similar results are observed.

In a scenario where $N$ is 20 (see Figure 3a), SPR has a drastic decrease in $\mathrm{BF}($ all $)$ over time, as compared to the other protocols. This is mostly due to the feature of SPR that allows a sensor node to use the same set of links constructing the shortest path in order to forward/relay packets. Eventually, this property heavily loads some nodes and causes them to die earlier. On the other hand, GLOBAL and CPL have better BF(all) than SPR, because both schemes distribute the network traffic through the gradient field constructed based on the cumulative path load of sensor nodes. However, in GLOBAL, since sensor nodes prefer the path excluding the most loaded node to

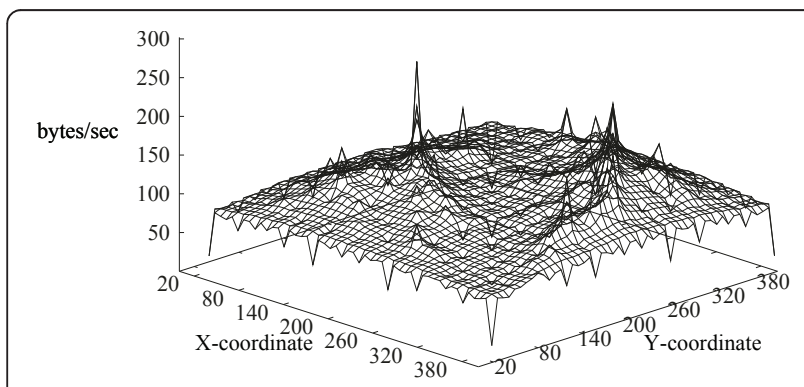

Figure 6 Load distribution in the $20 \times 20$ grid network with two sinks (Coordinates of sinks: $(120,120)$ and $(300,280)$ ). the least overloaded path as their hop distance to a sink decreases, GLOBAL cannot outperform CPL. GLOBAL outperforms SPR by $3.2 \%$, while CPL outperforms SPR by $12.2 \%$ at time $450 \mathrm{~s}$. Table 2 summarizes the performance gains that GLOBAL and CPL could achieve in terms of $\mathrm{BF}(\mathrm{all})$, as compared to SPR. Since the uneven traffic load distribution becomes more serious as the network scales, their gains compared to SPR are expected to increase with the network scale.

In order to analyze the impact of increasing a traffic rate on the $\mathrm{BF}(\mathrm{all})$ performance, we also measured the $\mathrm{BF}(\mathrm{all})$ value of the compared protocols using different $R$ values in the grid scenario where $N$ was set to 20 . As sensor nodes generate their traffic at a higher rate, sensor nodes located in hot spot regions become more overloaded, which makes the distribution of traffic load in the network unbalanced. In this respect, the effect of increasing a traffic rate is almost identical to that of increasing a network scale given a fixed traffic rate. We obtained similar simulation results with each other. Table 3 summarizes the performance gain that GLOBAL could achieve in terms of $\mathrm{BF}($ all $)$ as compared to the other protocols for different traffic rates.

Due to the characteristics of convergecast traffic to each sink, sensor nodes around sinks tend to be highly overloaded. Since the network lifetime is limited by the lifetime of the most overloaded node among them, it is important to evenly distribute the traffic load around sinks. Based on the reason, we also measured BF for 1hop neighbor nodes of sinks (denoted by BF(1-hop)). In GLOBAL, in order to enable sensor nodes to avoid paths including the most overloaded node, the gradient field is constructed by weighting the cumulative path load as well as the traffic load of the most overloaded node. On the other hand, since CPL creates it gradient field, based 


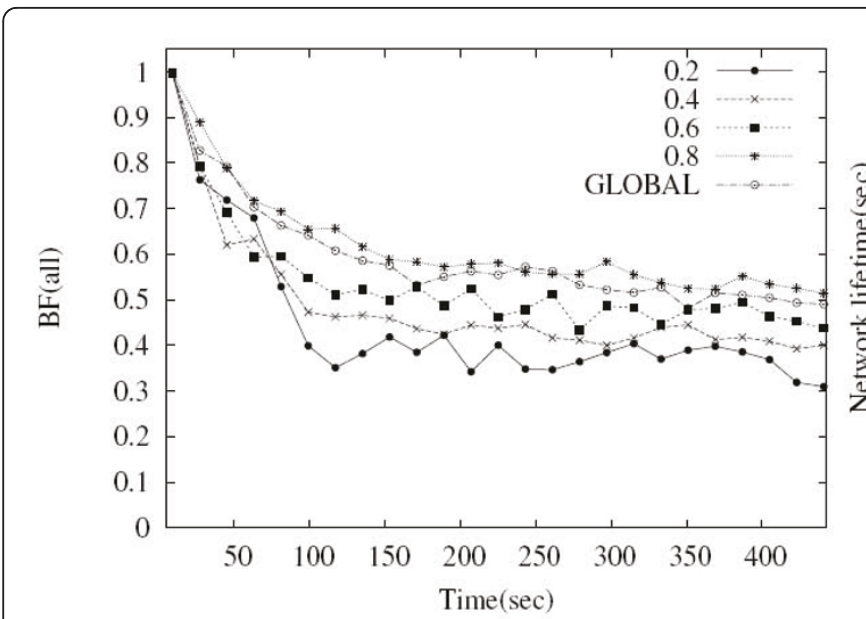

(a)

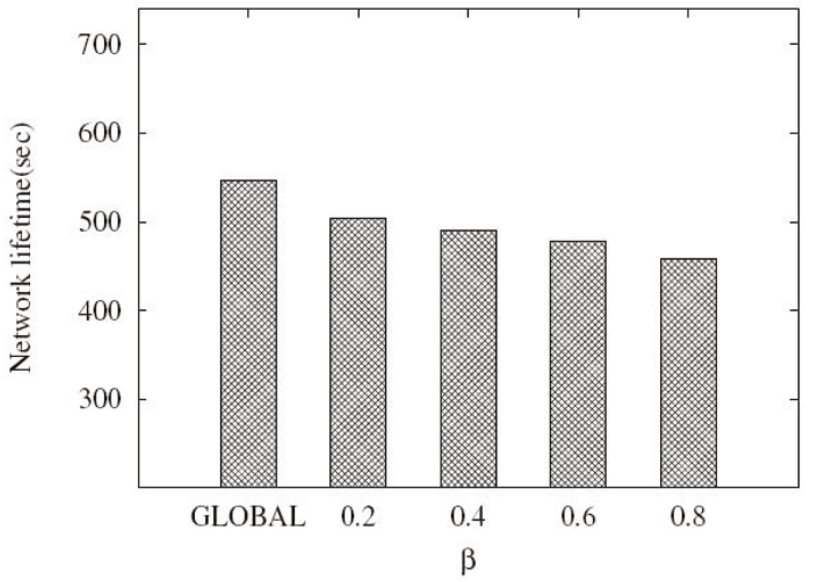

(b)

Figure 7 The performance of GLOBAL: $\mathrm{BF}($ all $)$ and network lifetime. a $\mathrm{BF}(\mathrm{all})$. b Network lifetime $\left(L T_{1}\right)$.

Table 2 The gain of GLOBAL and CPL for different network scales (w.r.t. BF(all))

\begin{tabular}{llll}
\hline Protocols & $\boldsymbol{N}=\mathbf{2 0}$ & $\boldsymbol{N}=\mathbf{3 0}$ & $\boldsymbol{N}=\mathbf{4 0}$ \\
\cline { 2 - 4 } & $\mathbf{4 0 0}$ nodes (\%) & $\mathbf{9 0 0}$ nodes $(\%)$ & $\mathbf{1 , 6 0 0}$ nodes $(\%)$ \\
\hline GLOBAL & 8.2 & 23.4 & 33.3 \\
CPL & 12.2 & 31.6 & 45.1 \\
\hline
\end{tabular}

Table 3 The gain of GLOBAL and CPL for different traffic rates (w.r.t. $B F(a l l)$ )

\begin{tabular}{llll}
\hline Protocols & $\boldsymbol{R}=\mathbf{5}(\%)$ & $\boldsymbol{R}=\mathbf{1 0}(\boldsymbol{\%})$ & $\boldsymbol{R}=\mathbf{1 5}(\%)$ \\
\hline GLOBAL & 8.2 & 13.7 & 18.2 \\
CPL & 12.2 & 21.3 & 31.2 \\
\hline
\end{tabular}

on only the cumulative path load, it cannot efficiently avoid using paths containing the most overloaded node. Therefore, CPL produces lower BF(1-hop) than GLOBAL, as shown in Figure 4. In particular, due to the superiority of GLOBAL's heuristic method to choose the weighting factor, GLOBAL keeps BF(1-hop) almost close to 1 from 0 to $450 \mathrm{~s}$ without significant decrease in $\mathrm{BF}$ (all) in case where $N$ is set to 20 (see Figure 4a). For all network scales, similar results are observed. In this scenario, GLOBAL outperforms SPR and CPL by 18.6 and $4.2 \%$, regarding $\mathrm{BF}(1$-hop) at time $450 \mathrm{~s}$, respectively.

Since the uneven traffic load distribution becomes more serious as the network scales, the GLOBAL's gain in $\mathrm{BF}$ is expected to increase with the network scale. Table 4 summarizes the performance gain that GLOBAL could achieve in terms of BF(1-hop), as compared to the other protocols. In addition, Table 5 summarizes the performance gain that GLOBAL could achieve in terms of $\mathrm{BF}(1$-hop) for different $R$ values in $20 \times 20$ grid
Table 4 The gain of GLOBAL for different network scales (w.r.t. BF(1-hop))

\begin{tabular}{llll}
\hline $\begin{array}{l}\text { Compared } \\
\text { protocols }\end{array}$ & $\boldsymbol{N}=\mathbf{2 0}$ & $\boldsymbol{N}=\mathbf{3 0}$ & $\mathbf{N = 4 0}$ \\
\cline { 2 - 4 } & $\begin{array}{lll}\mathbf{4 0 0} \text { nodes } \\
\mathbf{( \% )}\end{array}$ & $\begin{array}{l}\mathbf{9 0 0} \text { nodes } \\
\mathbf{( \% )}\end{array}$ & $\begin{array}{l}\mathbf{1 , 6 0 0} \text { nodes } \\
(\%)\end{array}$ \\
\hline SPR & 18.6 & 27.9 & 42.1 \\
$\mathrm{CPL}$ & 4.2 & 10.2 & 20.4 \\
\hline
\end{tabular}

Table 5 The gain of GLOBAL for different traffic rates (w.r.t. BF(1-hop))

\begin{tabular}{llll}
\hline Compared protocols & $\boldsymbol{R}=\mathbf{5}(\%)$ & $\boldsymbol{R}=\mathbf{1 0}(\%)$ & $\boldsymbol{R}=\mathbf{1 5}(\%)$ \\
\hline SPR & 18.6 & 25.7 & 30.2 \\
CPL & 4.2 & 8.6 & 18.3 \\
\hline
\end{tabular}

networks. As mentioned earlier, we observed similar simulation results to those with different network scales.

\section{C.2 Network lifetime}

Second, we measured the average network lifetime of the compared protocols after running above 50 runs. we define the network lifetime in two ways: $L T_{1}$ and $L T_{\%}$. Figure 8 a shows $L T_{1}$ as a function of network scale. As mentioned before, sensor nodes around sinks tend to be highly overloaded and the network lifetime is limited by the lifetime of the most overloaded node among them. As shown in Figure 8a, since GLOBAL shows the best $\mathrm{BF}$ (1-hop), it also has the best $L T_{1}$ as expected. In addition, we observe that the gain in $L T_{1}$ also increases with the network scale. Table 6 summarizes the performance gain that GLOBAL could achieve in terms of $L T_{1}$ as compared to the other protocols.

We also measure $L T_{\%}$ in $20 \times 20$ grid networks. Figure $8 \mathrm{~b}$ shows how $L T_{\%}$ changes by varying the $P$ 


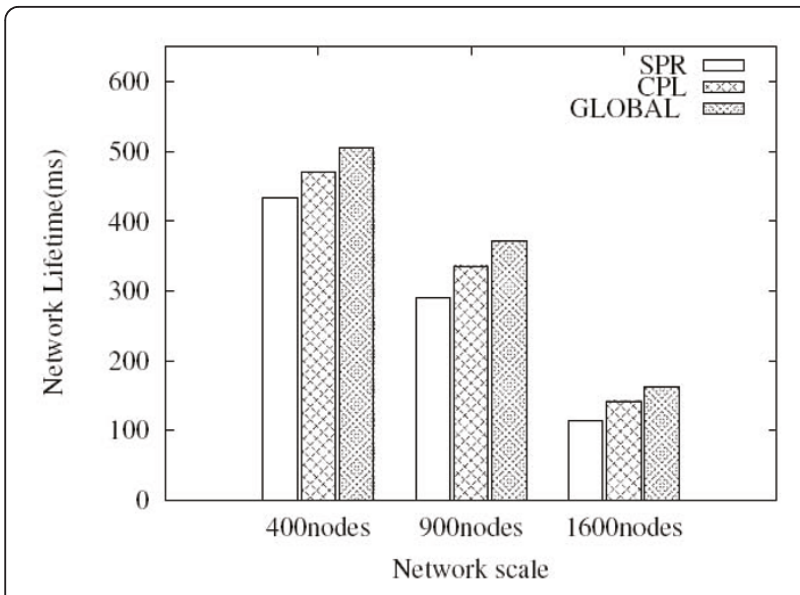

(a)

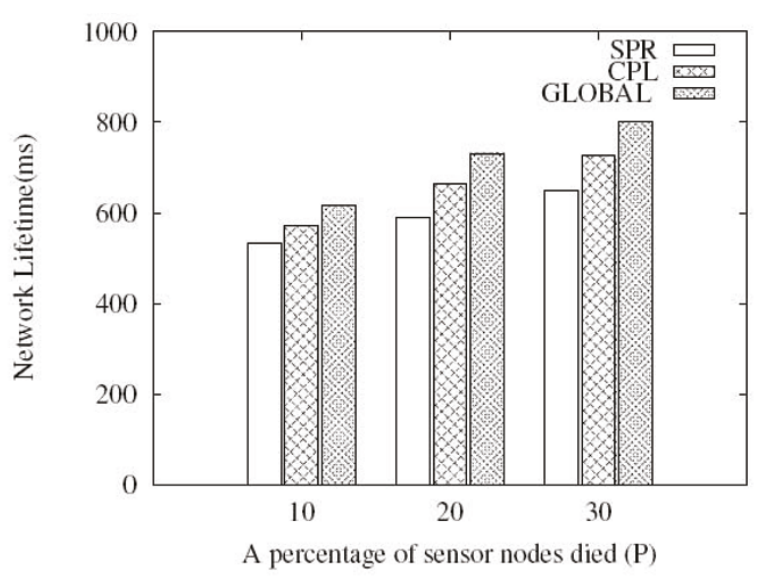

(b)

Figure 8 Performance comparison (w.r.t. Network lifetime). a $L T_{1}, \mathbf{b} L T \%$.

values. Whenever the most overloaded node becomes dead, one of sensor nodes which are still staying alive becomes the most overloaded node newly. Since the GLOBAL's gradient model reflects the traffic load of the most overloaded node which can be changed over time, GLOBAL efficiently allows sensor nodes to avoid the path including the most overloaded node even after some sensor nodes die. On the other hand, CPL cannot efficiently avoid using paths containing the most overloaded node. Therefore, GLOBAL keeps its high gain, regardless of the $P$ values as shown in Figure $8 \mathrm{~b}$. Table 7 summarizes the performance gain that GLOBAL could achieve in terms of $L T_{\%}$ as compared to the other protocols.

\section{C.3 PDR}

Third, we investigated PDR of each protocol. Figure 9 depicts PDR values at the time when the network lifetime is terminated. The unbalance of network traffic load causes a channel around overloaded regions to be congested, which leads to the increase in packet loss due to collisions and buffer overflow [12,17]. Hence, since SPR lacks the load-balancing functionality, it shows lower PDR performance than the other load-balancing protocols (i.e., GLOBAL, CPL). For example, in case where $N$ is set to 20, GLOBAL and CPL outperform SPR by 4.5 and $3.3 \%$, respectively. The degree of congestion around overloaded regions become obviously more severe as the

Table 6 The gain of GLOBAL (w.r.t. $L T_{1}$ )

\begin{tabular}{llll}
\hline Compared protocols & $\boldsymbol{N = 2 0}$ & $\boldsymbol{N}=\mathbf{3 0}$ & $\boldsymbol{N}=\mathbf{4 0}$ \\
\cline { 2 - 4 } & $\begin{array}{l}\mathbf{4 0 0} \text { nodes } \\
(\mathbf{\%})\end{array}$ & $\begin{array}{l}\mathbf{9 0 0} \text { nodes } \\
(\mathbf{\%})\end{array}$ & $\begin{array}{l}\mathbf{1 , 6 0 0} \text { nodes } \\
(\%)\end{array}$ \\
\hline SPR & 11.6 & 27.9 & 42.1 \\
CPL & 7.4 & 10.5 & 14.1 \\
\hline
\end{tabular}

network scales. As expected, both load-balancing protocols' gain in PDR increases with the network scale as shown in Figure 9a. For the same reason, the gain of PDR in both load-balancing protocols increases with the traffic rate as shown in Figure 9b. We also examined the causes of packet loss. As shown in Figure 10, most of packet loss results from buffer overflow, followed by packet collisions. Since sensor nodes cannot have an abundant storage to buffer packets in resource-constrained WSNs, our simulation set the buffer size to 10 packets.

As observed in previous simulation results, sensor nodes around sinks become highly overloaded and GLOBAL can balance the traffic load, especially around sinks. Therefore, GLOBAL allows more packets to reach sinks than CPL. That is, it shows better PDR than CPL. GLOBAL outperforms CPL by $3.3 \%$ in case where $N$ is set to 20 and the GLOBAL's gain in PDR increases with the network scale as shown in Figure 9. Tables 8 and 9 summarize the performance gain that GLOBAL could achieve in terms of PDR for different network scales and traffic rates, respectively.

\section{C.4 Average end-to-end delay}

Finally, we measured the average end-to-end delay of the compared protocols for different network scales and traffic rates. As shown in Figure 11, the average end-to-end delays of GLOBAL and CPL are lower than those of SRP. This is because both protocols prefer a path with light traffic load and thus reduce the queuing delay in the packet buffer. However, since GLOBAL requires a sensor

Table 7 The gain of GLOBAL (w.r.t. $L T_{\%}$ )

\begin{tabular}{llll}
\hline Compared protocols & $\boldsymbol{P}=\mathbf{1 0}(\%)$ & $\boldsymbol{P}=\mathbf{2 0}(\%)$ & $\boldsymbol{P}=\mathbf{3 0}(\%)$ \\
\hline SPR & 15.9 & 24.1 & 23.45 \\
CPL & 7.7 & 10.1 & 10.3
\end{tabular}




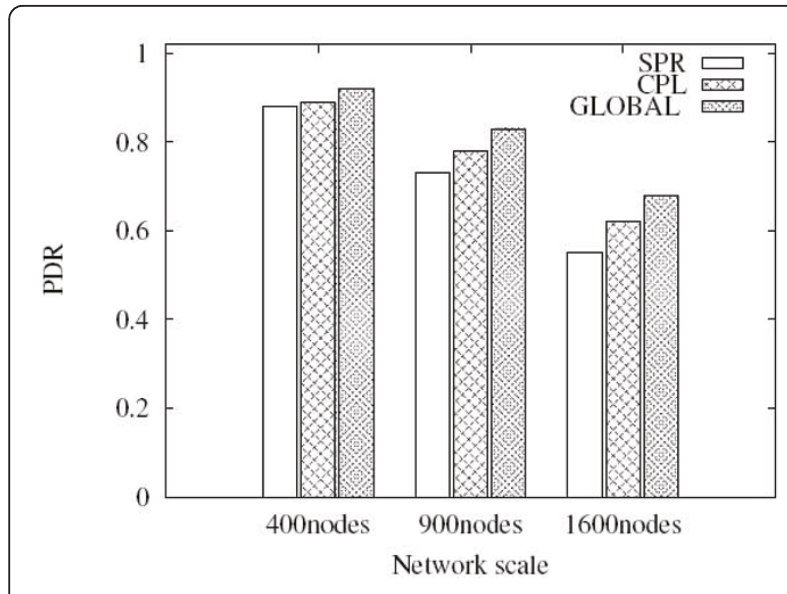

(a)

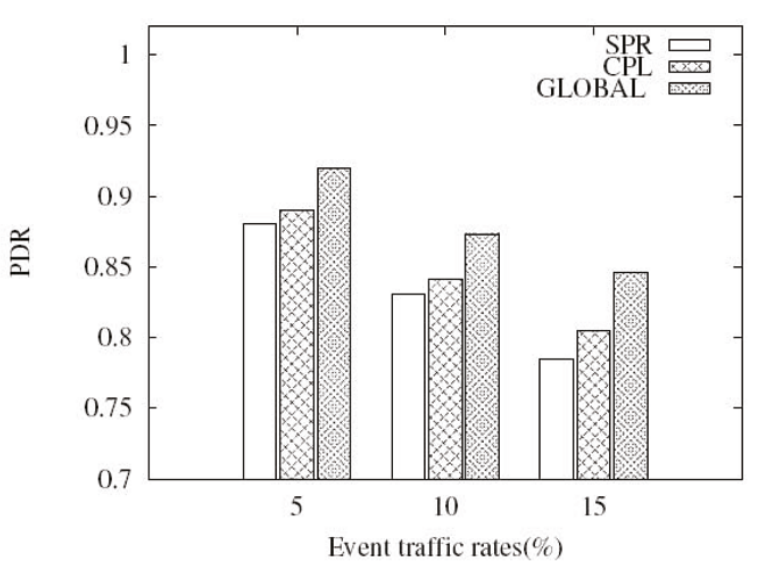

(b)

Figure 9 Performance comparison (w.r.t. PDR). a PDR for different network scales, b PDR for different traffic rates.

node to favor the path excluding the most loaded node as its hop distance to a sink decreases, the traffic load of the selected path may be higher than that of the least-loaded path. Therefore, GLOBAL has a slightly higher average end-to-end delay than CPL, but the difference is negligible. Since the traffic load of the path increases with the amount of traffic generated from the network, the GLOBAL's gain is expected to decrease with the network scale and traffic rate, as compared to CPL. Tables 10 and 11 summarize the performance gain that GLOBAL could achieve in terms of the average end-to-end delay for different network scales and traffic rates, respectively.

\section{C.5 Analysis of routing overhead}

Normalized routing load (NRL) is defined as the number of control packets transmitted per data packet delivered at the destination. NRL has been commonly used to evaluate the overhead of routing protocols. In the compared protocols, the ADV packet is the only control packet and sinks flood the ADV packet only once when a network is

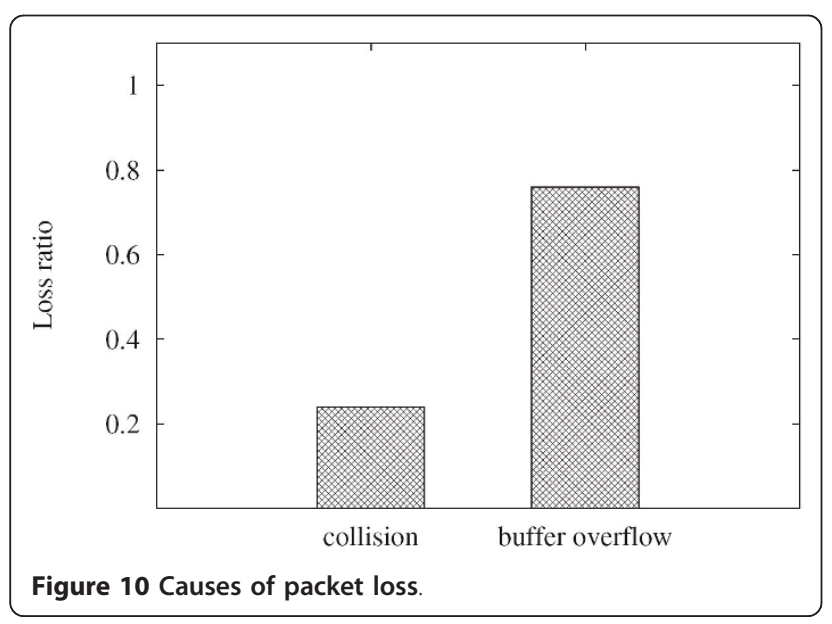

initialized. Therefore, they have almost the same NRL among each other. Unlike SPR, GLOBAL and CPL, however, require sensor nodes to piggyback control information on the periodic data packet. We therefore measured their byte-level control overhead, since NRL cannot reflect the control overhead caused by the piggybacking. In the compared protocols, the overhead is affected by three parameters: the size of information piggybacked on the periodic data packet, the period of the periodic data transmission, and the number of sensor nodes. Since we have fixed values for such parameters, their byte-level control over-head was measured numerically without simulations.

In GLOBAL, sensor nodes are allowed to piggyback the 5-byte information (i.e., hcnt, sum_redr, and max_redr) on their periodic data packet. On the other hand, CPL requires the 3-byte information (i.e., hcnt and sum_redr) to be piggybacked on the periodic data packet, and SPR does not require any piggybacking. Therefore, assuming that there are $N_{\text {sensor }}$ sensor nodes and they transmit $k$ periodic data packets per second, GLOBAL and CPL have the routing overhead of $5 \cdot N_{\text {sensor }} \cdot k$ bytes $/ s$ and $3 \cdot N_{\text {sensor }}$ $\cdot k$ bytes/s, respectively. In addition, the routing overhead caused by the ADV flooding is calculated to be $L_{\mathrm{ADV}}$. $N_{\text {sink }} \cdot N_{\text {sensor }}$ bytes, where $L_{\mathrm{ADV}}$ is the length of the ADV message in bytes and $N_{\text {sink }}$ is the number of sinks.

\section{Conclusion}

For the purpose of load balancing, the cumulative traffic load of a path is considered in existing gradient routing protocols. However, they have a critical drawback that a sensor node cannot efficiently avoid using the path with the most overloaded node. Only the information on cumulative path load is not enough to identify the path including the most overloaded node. Hence, we proposed a new gradient routing protocol (called GLOBAL). In 
Table 8 The gain of GLOBAL for different network scales (w.r.t. PDR)

\begin{tabular}{llll}
\hline $\begin{array}{l}\text { Compared } \\
\text { protocols }\end{array}$ & $\boldsymbol{N}=\mathbf{2 0}$ & $\boldsymbol{N}=\mathbf{3 0}$ & $\boldsymbol{N}=\mathbf{4 0}$ \\
\cline { 2 - 4 } & $\begin{array}{l}\mathbf{4 0 0} \text { nodes } \\
(\%)\end{array}$ & $\begin{array}{l}\mathbf{9 0 0} \text { nodes } \\
(\%)\end{array}$ & $\begin{array}{l}\mathbf{1}, 600 \text { nodes } \\
(\%)\end{array}$ \\
\hline SPR & 4.5 & 13.65 & 23.6 \\
CPL & 3.3 & 6.45 & 13.3 \\
\hline
\end{tabular}

Table 9 The gain of GLOBAL for different traffic rates (w. r.t. PDR)

\begin{tabular}{llll}
\hline Compared protocols & $\boldsymbol{R}=\mathbf{5}(\%)$ & $\boldsymbol{R}=\mathbf{1 0}(\%)$ & $\boldsymbol{R}=\mathbf{1 5}(\%)$ \\
\hline $\mathrm{SPR}$ & 4.5 & 5.18 & 8.4 \\
$\mathrm{CPL}$ & 3.3 & 4.1 & 5.3 \\
\hline
\end{tabular}

GLOBAL, each sensor node determines its gradient by weighting the cumulative load of a path and the most overloaded node over the path through a weight factor $\beta$. Using ns- 2 simulator, we explored the impact of $\beta$ on the GLOBAL performance and found that there exists a trade-off between the even distribution of traffic load and network lifetime. Based on simulation results, we introduced a heuristic method to choose $\beta$ in order to maximize network lifetime while minimizing deterioration in load-balancing performance. Through the proposed heuristic method, each sensor node is enabled to more favor paths excluding the most overloaded node as its shortest hop distance to a sink decreases. This makes the traffic load of sensor nodes around sinks effectively balanced while allowing sensor nodes far away from sinks to favor the least-loaded path. In addition, we also proposed an addressing-free forwarding strategy in order to avoid addressing overhead. Instead of using an address for forwarding packets, GLOBAL uses the gradient value as the next-hop node identifier.

Through ns-2 simulation, we conducted performance comparisons with other routing protocols in static grid networks: shortest path routing (SPR) and CPL, where $\mathrm{CPL}$ is a gradient routing using the cumulative path load only. We measured four metrics: balance factor, network lifetime, packet delivery ratio, and average endto-end delay. GLOBAL always achieves better performances than the others in terms of the former three metrics. In particular, assuming that the network lifetime is defined as the time from network deployment to the instant when one of any sensor nodes dies, GLOBAL shows the performance improvements in the network lifetime by 42.1 and $14.1 \%$ at a $40 \times 40$ grid network, as compared to SPR and CPL, respectively. This implies that GLOBAL can achieve its main goal of extending the network lifetime. However, GLOBAL has a slightly higher average end-to-end delay than CPL, but the difference is negligible. In this work, GLOBAL did not take the mobility of sensor nodes and sinks into account. Its performance degradation in dynamic networks with node mobility is unavoidable. Hence, its robustness against the frequent change of network topology will be improved in our future work.

\section{Endnotes}

a The cumulative traffic load information of the path is simply called as cumulative path load.

${ }^{\mathrm{b}} \mathrm{A}$ simple battery life indicator based solely on the battery voltage cannot provide high-resolution measurement. Resolution refers to the smallest quantity of energy that a system can measure. However, more sophisticated devices can measure their battery level

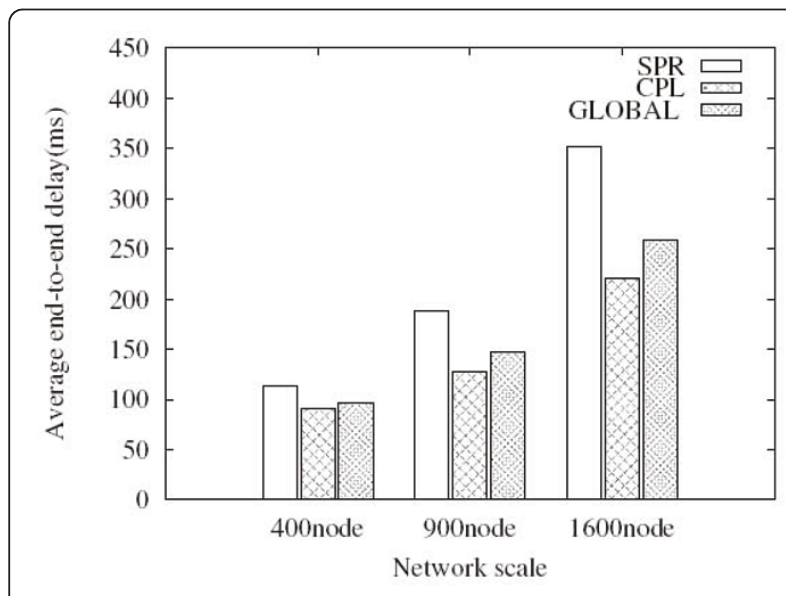

(a)

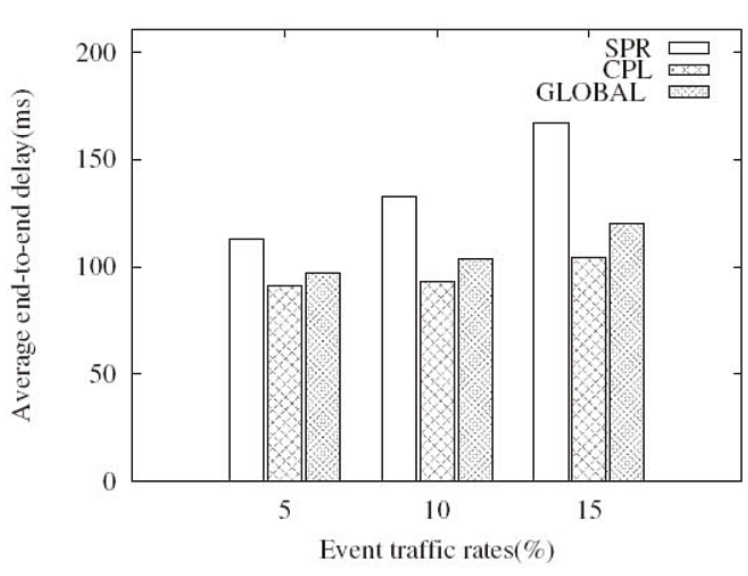

(b)

Figure 11 Performance comparison (w.r.t. Average end-to-end delay). a Average end-to-end delay for different network scales, b Average end-to-end delay for different traffic rates. 
Table 10 The gain of GLOBAL for different network scales (w.r.t. Average end-to-end delay)

\begin{tabular}{llll}
\hline $\begin{array}{l}\text { Compared } \\
\text { protocols }\end{array}$ & $\boldsymbol{N}=\mathbf{2 0}$ & $\boldsymbol{N}=\mathbf{3 0}$ & $\boldsymbol{N}=\mathbf{4 0}$ \\
\cline { 2 - 4 } & $\begin{array}{lll}\mathbf{4 0 0} \text { nodes } \\
(\%)\end{array}$ & $\begin{array}{l}\mathbf{9 0 0} \text { nodes } \\
(\%)\end{array}$ & $\begin{array}{l}\mathbf{1 , 6 0 0} \text { nodes } \\
(\%)\end{array}$ \\
\hline SPR & 14.2 & 21.8 & 26.5 \\
CPL & -6.5 & -14.4 & -17.5 \\
\hline
\end{tabular}

Table 11 The gain of GLOBAL for different traffic rates (w.r.t. Average end-to-end delay)

\begin{tabular}{llll}
\hline Compared protocols & $\boldsymbol{R}=\mathbf{5}(\%)$ & $\boldsymbol{R}=\mathbf{1 0}(\%)$ & $\boldsymbol{R}=\mathbf{1 5}$ (\%) \\
\hline SPR & 14.2 & 21.85 & 28.4 \\
CPL & -6.5 & -11.4 & -15.22 \\
\hline
\end{tabular}

based on the average of the energy drained from the battery. As mentioned in [19], high-resolution battery measuring can be performed using either hardware support, as in systems like Quanto [23] and iCount [24], or using software monitoring, as in Pixie [25] and a resolution of $0.1 \mu \mathrm{J}$ can be achieved.

${ }^{c}$ Ye et al. [4] proposed an efficient backoff-based gradient field establishment algorithm, which attempts to find the optimal costs of all nodes to the sink with the overhead of one packet per node. Although GLOBAL currently utilizes the naive flooding-based gradient field establishment algorithm, it can simply adopt the backoff-based one. The performance improvement issue in the gradient field establishment algorithm is out of scope of this paper.

${ }^{a}$ Assuming that sinks and sensor nodes have no mobility, the evaluation of performance in dynamic networks is beyond the scope of this work.

${ }^{\mathrm{e}}$ net-diameter is a system parameter. The calculation of net-diameter is out of scope of this paper.

\section{Author details}

${ }^{1}$ Graduate School of Electrical Engineering and Computer Science, Kyungpook National University, Daegu, Korea ${ }^{2}$ School of Computer Science and Engineering, College of IT Engineering, Kyungpook National University, Daegu, Korea

\section{Competing interests}

The authors declare that they have no competing interests.

Received: 15 June 2010 Accepted: 1 September 2011 Published: 1 September 2011

\section{References}

1. M Perillo, Z Cheng, W Heinzelman, Strategies for Mitigating the Sensor Network Hot Spot Problem, in Proceedings of MobiQuitous (July 2005)

2. D Puccinelli, M Haenggi, Arbutus: Network-Layer Load Balancing for Wireless Sensor Networks, in Proceedings of IEEE WCNC (April 2008)

3. I Slama, B Jouaber, D Zeghlache, Energy Efficient Scheme for Large Scale Wireless Sensor Networks with Multiple Sinks, in Proceedings of IEEE WCNC (March 2008)
4. F Ye, A Chen, S Lu, L Zhang, A Saclable Solution to Minimum Cost Forwarding in Large Sensor Networlks, in Proceedings of IEEE ICCCN (October 2001)

5. JN Al-Karaki, AE Kamal, Routing techniques in wireless sensor networks: a survey. IEEE Wirel Commun. 11(6), 6-28 (2004). doi:10.1109/ MWC.2004.1368893

6. P Huang, $\mathrm{H}$ Chen, G Xing, Y Tan, SGF: a state-free gradient-based forwarding protocol for wireless sensor networks. ACM Trans Sen Netw. 5(2), 1-25 (2009)

7. A Basu, A Lin, S Ramanathan, Routing Using Potentials: A Dynamic TrafficAware Routing Algorithm, in Proceedings of ACM SIGCOMM (August 2003)

8. RH Cheng, SY Peng, C Huang, A Gradient-Based Dtnamic Load Balance Data Forwarding Method for Multi-Sink Wireless Snesor Networks, in Proceedings of IEEE APSCC (December 2008)

9. H Liu, ZL Zhang, J Srivastava, V Firoiu, PWave: A Multi-Source Multi-Sink Anycast Routing Framework for Wireless Sensor Networks, in Proceedings of IFIP Networking (May 2007)

10. RC Shah, JM Rabaey, Energy Aware Routing for Low Energy Ad Hoc Sensor Networks, in Proceedings of IEEE WCNC (March 2002)

11. J Suhonen, M kuorilehto, M Hannikainen, TD Hamalainen, Cost-Aware Dynamic Routing Protocol for Wireless Sensor Networks-Design and Prototype Experiments, in Proceedings of IEEE PIMRC (September 2006)

12. M Fyffe, M Sun, X Ma, Traffic-Adapted Load Balancing in Sensor Networks Employing Geographic Routing, in Proceedings of IEEE WCNC (March 2006)

13. S Singh, M Woo, CS Raghavendra, Power-Aware Routing in Mobile Ad Hoc Networks, in Proceedings of ACM/IEEE MobiCom (October 1998)

14. R Draves, J Padhye, B Zill, Routing in Multi-Radio, Multi-Hop Wireless Mesh Networks, in Proceedings of ACM/IEEE MobiCom (September 2004)

15. $F$ Ye, G Zhoung, S Lu, L Zhang, GRAdient broadcast: a robust data delivery protocol for large scale sensor networks. Wirel Netw. 11(3), 285-298 (2005). doi:10.1007/s11276-005-6612-9

16. T Roosta, WC Liao, WC Teng, S Sastry, Testbed Implementation of a Secure Flooding Time Synchronization Protocol, in Proceedings of IEEE WCNC (March 2008)

17. K Wu, J Harms, Load-Sensitive Routing for Mobile Ad Hoc Networks, in Proceedings of IEEE ICCCN (October 2001)

18. D Kim, JJ Garcia-Luna-Aceves, K Obraczka, J-C Cano, P Manzoni, Routing mechanisms for mobile ad hoc networks based on the energy drain rate. IEEE Trans Mob Comput. 2(2), 161-173 (2003). doi:10.1109/ TMC.2003.1217236

19. GW Challen, J Waterman, M Welsh, Idea: Integrated Distributed Energy Awareness for Wireless Sensor Networks, in Proceedings of ACM MobiSys (June 2010)

20. P Basu, J Redi, Effect of Overhearing Transmissions on Energy Efficiency in Dense Sensor Networks, in Proceedings of IEEE IPSN (April 2004)

21. H Dai, R Han, A Node-Centric Load Balancing Algorithm for Wireless Sensor Networks, in Proceedings of IEEE GLOBECOM (December 2003)

22. Y Chen, Q Zhao, On the lifetime of wireless sensor networks. IEEE Commun Lett. 9, 976-978 (2005). doi:10.1109/LCOMM.2005.11010

23. R Fonseca, P Dutta, P Levis, I Stoica, Quanto: Tracking Energy in Networked Embedded Systems, in Proceedings of OSDI (December 2008)

24. P Dutta, M Feldmeier, J Paradiso, D culler, Energy Metering for Free: Augmenting Switching Regulators for Real-Time Monitoring, in Proceedings of IEEE IPSN (April 2008)

25. K Lorincz, B Chen, J Waterman, G Werner-Allen, M Welsh, Resource Aware Programming in the Pixie os, in Proceedings of ACM SenSys (November 2008)

doi:10.1186/1687-1499-2011-85

Cite this article as: Yoo et al:: A scalable multi-sink gradient-based routing protocol for traffic load balancing. EURASIP Journal on Wireless Communications and Networking 2011 2011:85. 